\title{
Análisis de las alternativas de los migrantes mexicanos en Estados Unidos de América para atender sus problemas de salud
}

\author{
Gustavo Nigenda, PhD,(') José Arturo Ruiz-Larios, Lic en Soc, (I) Rosa María Bejarano-Arias, Lic en Soc,(l) \\ Jacqueline Elizabeth Alcalde-Rabanal, MSP,(I) Pastor Bonilla-Fernández, MSc.(I)
}

\begin{abstract}
Nigenda G, Ruiz-Larios JA, Bejarano-Arias RM, Alcalde-Rabanal JE, Bonilla-Fernández P. Análisis de las alternativas de los migrantes mexicanos en Estados Unidos de América para atender sus problemas de salud.
\end{abstract} Salud Publica Mex 2009;5 I:407-4I6.

\section{Resumen}

Objetivo. Identificar las alternativas ideadas por los migrantes mexicanos para acceder a los servicios de salud en Estados Unidos de América y México. Material y métodos. Estudio etnográfico en cinco estados, seleccionados por su dinámica migratoria. Se realizaron grupos focales y entrevistas a profundidad con usuarios y prestadores de servicios. Resultados. Se identificaron seis formas de atención utilizadas de acuerdo con la gravedad de la enfermedad:a) automedicación; b) consulta telefónica con familiares; c) uso de servicios privados; d) traslado a ciudades fronterizas; e) retorno al lugar de nacimiento;f) atención durante visitas estacionales al lugar de origen. Discusión. Las alternativas empleadas por los migrantes para acceder a los servicios de salud se explican por las circunstancias del contexto de migración y la falta de opciones formales de atención. Es fundamental la aplicación de una política pública para la protección de la salud de estos ciudadanos en ambos lados de la frontera.

Palabras clave: migrantes; alternativas de atención a la salud; servicios de salud; México
Nigenda G, Ruiz-Larios JA, Bejarano-Arias RM,

Alcalde-Rabanal JE, Bonilla-Fernández $P$.

Strategies by Mexican migrants in the USA

to seek health care.

Salud Publica Mex 2009;5 I:407-4I6.

\section{Abstract}

Objective. To identify the strategies used by Mexican migrants to access health services in the U.S. and Mexico. Materials and Methods. Ethnographic study in five Mexican states, selected by their migration dynamics. A series of focus groups and in-depth interviews were conducted with health services users and providers. Results. Six strategies were identified and are used according to the severity of the disease: a) self-medication, b) telephone consultation with relatives, c) utilization of private services, d) travel to border towns, e) return to place of birth, f) getting medical care during their visits to places of origin. Discussion. The health care options used by Mexican migrants and their families are defined according to events related to the migration process. The implementation of public policies to protect the health of migrants on both sides of the border is critical.

Key words: migrants; health care; health services; Mexico

(I) Centro de Investigaciones en Sistemas de Salud, Instituto Nacional de Salud Pública. Cuernavaca, Morelos. México.

Fecha de recibido: 24 de septiembre de 2008 - Fecha de aprobado: 7 de julio de 2009

Solicitud de sobretiros: Dr. Gustavo Nigenda López. Dirección de Innovación de Servicios y Sistemas de Salud. Centro de Investigaciones en Sistemas de Salud. Instituto Nacional de Salud Pública. Av. Universidad 655, col. Santa María Ahuacatitlán. 62100, Cuernavaca, Morelos, México.

Correo electrónico: gnigenda@insp.mx 
T a migración mexicana hacia Estados Unidos de $\amalg$ América (EUA) tiene sus raíces en la segunda mitad del siglo XIX y, si bien ha sido una constante desde hace más de una centuria y media, el fenómeno se ha intensificado de modo considerable en las últimas tres décadas. La mayor parte de los movimientos poblacionales en todas las regiones geográficas del mundo es consecuencia de la búsqueda de mejores condiciones de vida. ${ }^{1}$ La migración mexicana hacia EUA se ha generado por la persistente demanda de mano de obra en los sectores agrícola, industrial y de servicios de esa nación, por la considerable diferencia salarial entre ambas economías y el intenso ritmo de crecimiento demográfico de la población mexicana en edad laboral, así como la insuficiente dinámica de la economía nacional para absorber el excedente de fuerza de trabajo y la tradición migratoria de diversas regiones del país. ${ }^{2}$

En el año 2000 se calculó la migración acumulada a EUA en 9200000 de mexicanos y para el año 2006 la cantidad aumentó a 11600 000. Estas cifras representan un incremento anual de 400000 personas. $^{3}$ De los casi 12000000 de migrantes, sólo 43\% posee documentos migratorios y por lo tanto tiene la posibilidad de insertarse formalmente en el mercado laboral. Los indocumentados representan el restante $56.4 \% .4$ Prentice y colaboradores notificaron que en el año 1965, 69\% de los inmigrantes indocumentados en Los Ángeles no se encontraba asegurado $^{5}$ y entre 1998 y 2003 este grupo representó hasta $86 \%$ del crecimiento de la población no asegurada. ${ }^{6}$ Evidencias internacionales muestran que los migrantes tienen mayor riesgo a enfermar y morir en el periodo inmediato posterior a la migración, respecto del resto de la población del país receptor. ${ }^{7}$ Los trabajadores migrantes tienen más posibilidades de sufrir accidentes ${ }^{8}$ y el estrés que se origina posterior a la migración se relaciona con elevadas tasas de suicidio. ${ }^{9}$ Estos fenómenos pueden vincularse con la falta de aseguramiento de la población migrante que lleva a la postergación de la atención médica, el agravamiento de los problemas crónicos y, en algunos casos, la repatriación forzosa ante la enfermedad. ${ }^{10}$

La gran mayoría de los migrantes mexicanos en EUA mantiene vínculos estrechos con su país. El hecho de que hasta $27 \%$ tenga hijos con residencia en México es testimonio de la cercanía de estos vínculos y es probable que la mayor parte de los migrantes mantenga a sus dependientes ancianos y a otros miembros de la familia extensa a través de remesas, dado que hasta $85 \%$ de ellos las envía en forma periódica. ${ }^{11}$ Según el Banco de México, en 2006 las remesas ascendieron a 23053 millones de dólares. ${ }^{12}$ Durante el periodo de 2002 a 2007 ese monto de divisas representó la segunda fuente externa de ingresos para la economía nacional.
El objetivo del presente trabajo fue identificar y describir las alternativas ideadas por los migrantes mexicanos para atender sus problemas de salud, tanto en EUA como en México. Este propósito forma parte de un estudio más amplio enfocado sobre todo en la identificación del papel potencial del Seguro Popular de Salud en la atención a familias con miembros migrantes en EUA. Se exponen tres escenarios o situaciones posibles, ante los cuales los migrantes definen alternativas que pueden considerarse de uso común en estas poblaciones.

\section{Material y métodos}

Se llevó a cabo un estudio transversal con componente de encuesta y estudio etnográfico con muestreo por conveniencia $^{13}$ en cinco estados de la República mexicana, seleccionados a partir de indicadores de dinámica migratoria. Esta selección se sustentó en los cálculos que realizó el Consejo Nacional de Población (CONAPO) a partir del XII Censo General de Población y Vivienda 2000. ${ }^{14}$ Se eligieron cuatro entidades que pertenecen a la región histórica de la migración (Guanajuato, Michoacán, San Luis Potosí, Zacatecas e Hidalgo, que forma parte de los estados emergentes en el fenómeno migratorio. ${ }^{15}$ En cada una de las cinco entidades se seleccionaron los tres municipios con mayor grado de expulsión de población (cuadro I).

Para la recopilación de la información notificada en este artículo se emplearon dos técnicas cualitativas: grupos focales con usuarios de servicios y entrevistas a profundidad con informantes clave. De modo inicial se estableció contacto con autoridades de las secretarías de salud de las entidades seleccionadas, a fin de precisar las poblaciones cubiertas por el Seguro Popular. Con posterioridad se seleccionaron las unidades de primer nivel de atención a visitar, así como el personal operativo a entrevistar. La selección del personal operativo se realizó a partir de su disponibilidad en el momento de la visita a la unidad de salud por parte del equipo de investigación. Antes del levantamiento de información en los estados seleccionados se diseñaron y ensayaron los instrumentos.

La selección de los familiares de los migrantes se efectuó directamente en las salas de espera de las unidades de primer nivel de atención seleccionadas, en donde también se entrevistó de manera informal a los migrantes que se encontraban de modo temporal en México. El criterio de selección fue la afiliación al Seguro Popular de Salud y la existencia de algún familiar que residiera en Estados Unidos. La participación de los informantes fue voluntaria. A cada uno de ellos se le entregó y leyó la carta de consentimiento informado 


\section{Cuadro I \\ CONDICIÓN SOCIODEMOGRÁFICA DE LOS ESTADOS Y MUNICIPIOS SELECCIONADOS, 2005}
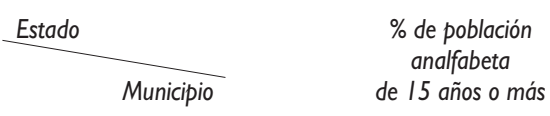

$\%$ de ocupantes de viviendas con piso de de 15 años o más

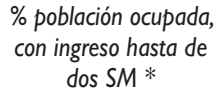

\% población ocupada con ingreso hasta de dos $S M *$

\section{$\%$ de hogares que reciben remesas}

\section{$\%$ de ocupantes en vivienda sin agua entubada}

\begin{tabular}{|c|c|c|c|c|c|}
\hline Guanajuato & 10.4 & 8.9 & 44.6 & 9.2 & 6.1 \\
\hline Acámbaro & 12.8 & 5.9 & 56.6 & 20.9 & 3.4 \\
\hline Huanímaro & 10.9 & 4.8 & 68.9 & 30.9 & 0.7 \\
\hline Santiago Maravatío & 18.1 & 5.1 & 57.1 & 27.4 & 0.06 \\
\hline Hidalgo & 12.8 & 12.7 & 61.6 & 5 & 12.2 \\
\hline Ixmiquilpan & 11.5 & 15.2 & 62.2 & 19.3 & 7.9 \\
\hline Pacula & 24.3 & 27.6 & 79.1 & 24.8 & 78.7 \\
\hline Tasquillo & 14.8 & 13.6 & 66.6 & 20.8 & 4.1 \\
\hline Michoacán & 12.5 & 15.6 & 55.7 & 11.3 & 9.9 \\
\hline Cotija & 17.6 & 8.5 & 56 & 23 & 9.6 \\
\hline Madero & 21.2 & 40.3 & 75 & 11.8 & 37.1 \\
\hline Zinapécuaro & 14.4 & 12.1 & 70.3 & 14.2 & 18.8 \\
\hline San Luis Potosí & 13.2 & 19.8 & 73.5 & 8.2 & 16.9 \\
\hline Rayón & 16.5 & 26.8 & 71.7 & 29.4 & 29.5 \\
\hline San Nicolás Tolentino & 19.9 & 20.7 & 77.9 & 28.5 & 33.5 \\
\hline Villa Juárez & 16.5 & 29.5 & 77.9 & 37 & 5.9 \\
\hline Zacatecas & 7.2 & 6.2 & 54.2 & 13 & 6.7 \\
\hline Mezquital del Oro & 10.3 & 18.6 & 54.6 & 20.4 & 39.4 \\
\hline Río Grande & 6.5 & 4.9 & 52.3 & 24.6 & 1.6 \\
\hline Villa Hidalgo & 13.3 & 13.4 & 70.1 & 18 & 2.5 \\
\hline Nacional & 8.3 & 11.4 & 43.3 & 4.3 & 10.1 \\
\hline
\end{tabular}

* Dos salarios mínimos al día, en el año 2008, eran equivalentes a 105.18 pesos moneda nacional. El estudio inició en junio del 2005 y concluyó en abril de 2008 Fuentes:

I. CONAPO, Índices de marginación por entidad federativa, 2005. Disponible en: http//www.conapo.gob.mx/publicaciones/margina 2005/AnexoA.pdf 2. CONAPO, 2002

aprobada por la comisión de ética del Instituto Nacional de Salud Pública.

En total se realizaron 14 grupos focales en cinco estados que contaron con la participación de 118 personas, con un promedio de ocho por grupo y con una duración promedio de 60 minutos. Hasta $84.4 \%$ de los participantes correspondió a mujeres y $15.6 \%$ a hombres, con un promedio de 41 años de edad; $73 \%$ se dedicaba al hogar y no tenía ingresos propios (cuadro II).

Se llevaron a cabo 34 entrevistas a profundidad con personal de salud y se indagó acerca del Seguro Popular, el acceso a servicios de salud de los migrantes y sus familias y las formas en que los migrantes enfrentan los problemas de salud. Las entrevistas tuvieron una duración promedio de 50 minutos.

Se utilizó el programa Atlas Ti para procesar la información obtenida de las entrevistas y los grupos focales. El procedimiento se inició con la definición de una lista de 48 códigos, todos ellos relacionados con los temas relativos a salud, migración e implementación del SPS. Cada uno de estos códigos representó un tema específico que permitió identificar, en el análisis de las transcripciones, aspectos relevantes en las opiniones de los informantes. 
Cuadro II

Perfil de los participantes en los grupos focales por estado

\begin{tabular}{|c|c|c|c|c|c|c|c|c|c|c|c|}
\hline \multirow{3}{*}{ Estado } & \multirow{3}{*}{$\begin{array}{c}\text { No. } \\
\text { Participantes }\end{array}$} & \multicolumn{5}{|c|}{ Grado de escolaridad (porcentajes) } & \multicolumn{4}{|c|}{ Ocupación (porcentajes) } & \multirow{3}{*}{$\begin{array}{l}\text { Porcentajes } \\
\text { afiliados } \\
\text { al SPS } 3 \\
2006\end{array}$} \\
\hline & & \multirow{2}{*}{$\begin{array}{c}\text { Sin } \\
\text { escolaridad }\end{array}$} & \multirow{2}{*}{$\begin{array}{c}\text { con } \\
\text { primaria }\end{array}$} & \multirow{2}{*}{$\begin{array}{c}\text { con } \\
\text { secundaria }\end{array}$} & \multirow{2}{*}{$\begin{array}{c}\text { con } \\
\text { bachillerato }\end{array}$} & \multirow{2}{*}{$\begin{array}{c}\text { con } \\
\text { licenciatura }\end{array}$} & \multirow{2}{*}{ Hogar } & \multicolumn{3}{|c|}{ Sector económico } & \\
\hline & & & & & & & & Primario & Secundario & Terciario & \\
\hline Guanajuato & 21 & 28.5 & 52.3 & 9.8 & 4.7 & 4.7 & 66 & 14 & 5 & 15 & 76.2 \\
\hline Hidalgo & 29 & 10.5 & 48.2 & 27.6 & 13.7 & 0 & 72 & 14 & 4 & 10 & 3.4 \\
\hline Michoacán & 25 & 4 & 48 & 16 & 20 & 12 & 60 & 8 & 4 & 28 & 36.0 \\
\hline SanLuis Potosí & 28 & 28 & 32.1 & 39.3 & 21.4 & 3.6 & 3.6 & 75 & 17.8 & 3.6 & 3.6 \\
\hline Zacatecas & 15 & 15 & 20 & 40 & 40 & 0 & 0 & 93 & 7 & 0 & 0 \\
\hline \multicolumn{12}{|c|}{$\begin{array}{l}\text { Fuente: } \\
\text { I. CONAPO, Índices de marginación por entidad federativa, 2005. Disponible en: http//www.conapo.gob.mx/publicaciones/margina 2005/AnexoA.pdf } \\
\text { 2. CONAPO, } 2002 \\
\text { 3. SSA. Sistema de Protección Social en Salud. Disponible en: http://sextoinforme.fac.presidencia.gob.mx/docs/anexo/pdf/p I23.pdf }\end{array}$} \\
\hline
\end{tabular}

Una vez codificadas las entrevistas, se efectuaron cruces de códigos para obtener familias que permitieran generar informes temáticos conformados por testimonios de diferentes informantes.

El estudio arrancó en junio del año 2005. El trabajo de campo comenzó en mayo del 2006 a partir de la validación de las comisiones de Ética e Investigación del Instituto Nacional de Salud Pública. El levantamiento de toda la información del estudio concluyó en abril de 2007. El proyecto en su conjunto finalizó en abril del año 2008.

\section{Resultados}

\section{Semejanzas y diferencias entre la población migrante de los cinco estados}

En las cinco entidades incluidas en el estudio, la información recabada señala que todos presentan un alto grado de intensidad migratoria; Zacatecas tiene el mayor porcentaje de hogares que reciben remesas, a diferencia de Oaxaca, donde a pesar de que el número de migrantes es alto, el porcentaje de hogares receptores de remesas es bajo.

Los testimonios recopilados marcan diferencias en los patrones de comportamiento de los migrantes según su lugar de origen. En efecto, los migrantes de Guanajuato, Michoacán y Zacatecas tienen más oportunidades de regresar a sus lugares de origen o a las ciudades fronterizas sin afrontar riesgo elevado de ser detenidos por las autoridades migratorias de EUA. En este sentido, juegan un papel importante las redes de familiares, amigos y las organizaciones de la sociedad civil que entre sus actividades se encuentra trasladar migrantes al otro lado de la frontera, en condiciones más seguras. Estas redes y organizaciones se ubican en ambos lados de la frontera.

Por su parte, los migrantes de Hidalgo y San Luis Potosí tienen menores opciones para apoyarse en este tipo de redes, por lo cual sus viajes a las ciudades mexicanas fronterizas en búsqueda de atención médica o medicamentos son menos frecuentes por el riesgo de enfrentar a la policía migratoria durante el cruce de la frontera con EUA.

\section{Utilización de tres escenarios y seis estrategias de búsqueda y atención para la salud de los migrantes}

Para tomar la decisión de acudir a los servicios de salud en México o EUA, el migrante lleva a cabo en primer lugar una valoración de la gravedad de la enfermedad y la fecha tentativa de retorno a su localidad de origen. Cuanto más próxima está la fecha de regreso a su comunidad, menor posibilidad existe de acudir a los servicios de salud en EUA. En general, se han identificado seis estrategias en que los migrantes mexicanos ponen en práctica cuando enfrentan algún problema de salud. Dichas modalidades se han ordenado en tres escenarios posibles. 
Primer escenario: atención médica de migrantes enfermos en EUA

En este escenario, además de la valoración de la gravedad de la enfermedad, se analiza la posibilidad de estar incapacitado para trabajar, el costo de los servicios privados al no contar con aseguramiento, el desconocimiento del idioma inglés y el contacto con el profesional de salud, lo cual incrementa el temor de ser denunciado a las autoridades migratorias. Tras la valoración de los aspectos señalados, se han identificado tres estrategias que los migrantes desarrollan para resolver su problema de salud en EUA y que no son mutuamente excluyentes (figura 1).

Estrategia 1: remedios caseros o automedicación

Cuando la enfermedad no es grave, se opta por los remedios caseros o la automedicación. Si no se dispone de medicamentos o remedios caseros, y si existe una buena comunicación con familiares en México, se solicita el envío ya sea de fármacos o insumos. En otros casos, el migrante o sus familiares llevan consigo medicamentos al momento de ingresar a territorio estadounidense.

Un participante de un grupo focal, con familiares que trabajaban en EUA, señaló:

Si está de ilegal y se enferman no van al doctor, tiene miedo de que lo regresen (...) prefieren curarse allá como sea (GP: P12)

Por su parte, el médico de un centro de salud afirmó:

Los que vienen y ya se van de regreso en lugar de llevar recuerdos, llevan cajas de medicamentos y allá se están autorrecetando, prácticamente, esos los encontramos a diario (P:10)

Estrategia 2: consulta telefónica con familiares (prescripción y envío de medicamentos desde México)

Cuando los remedios caseros no dan el resultado esperado, el migrante puede optar por comunicarse vía

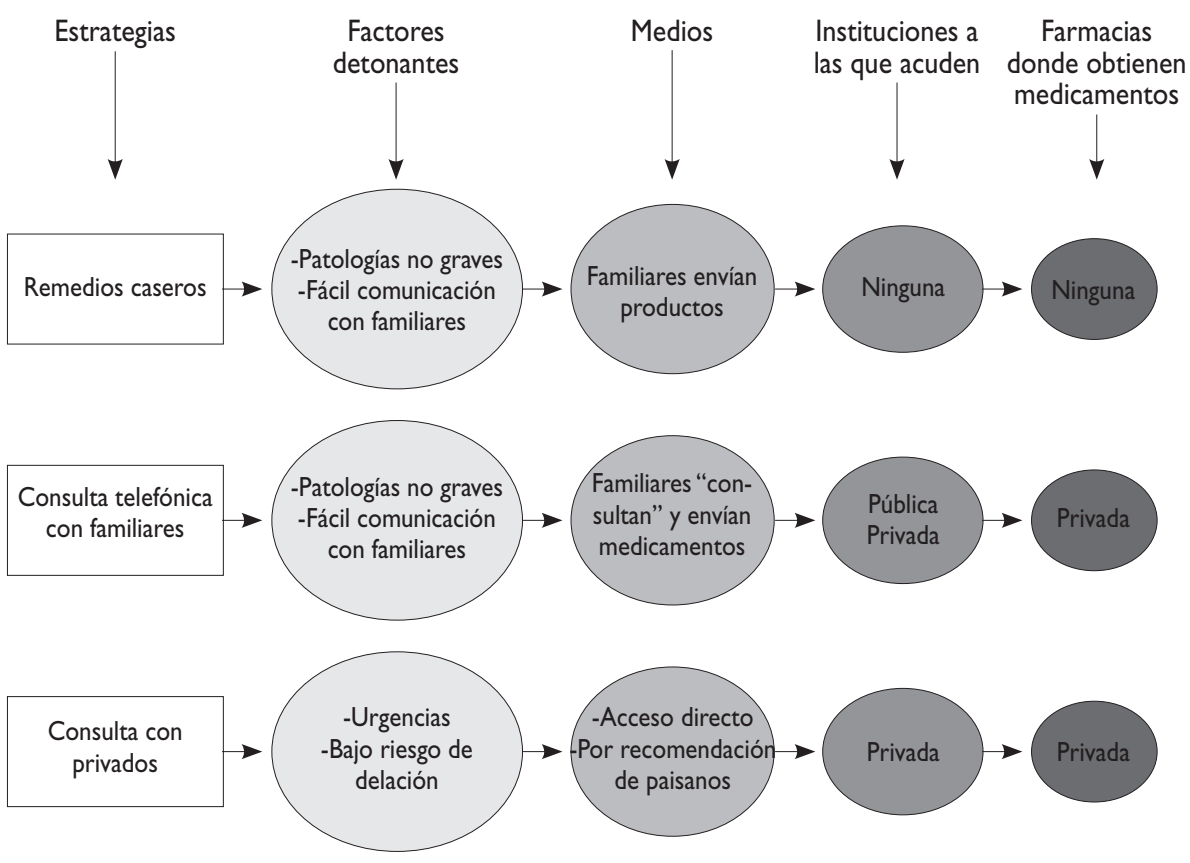

Fuente: elaboración propia a partir de los datos del trabajo de campo realizado entre mayo de 2006 y abril de 2007

El estudio inició en junio del 2005 y concluyó en abril del 2008

Figura I. Primer escenario. Atención médica de migrantes enfermos en los EUA 
telefónica con un miembro de la familia para notificarle los síntomas que presenta. La persona receptora de la información acude a una unidad médica pública o privada, para "consultar" al médico respecto de los síntomas que manifiesta sufrir el migrante en EUA. Con esta información el médico formula un diagnóstico aproximado y prescribe algunos fármacos. Más adelante, el familiar surte la receta, las más de las veces en una farmacia privada* y envía los medicamentos y las indicaciones clínicas por paquetería al domicilio del migrante en EUA.

Uno de los médicos entrevistados describió la siguiente situación:

Los migrantes se comunican por teléfono y llegan los familiares y dicen "fijese que mi esposo tiene un dolor de cabeza, tiene esto, tiene lo otro, ¿qué le mando? y a uno lo comprometen porque no es ético mandar así el medicamento pero como llegan con su caja de medicamento y diciendo, mire doctor le voy a mandar esto, ¿cómo ve? Nosotros sólo les decimos, sí mándele ése (P:11)

\footnotetext{
* El médico de un centro de salud público reconoció que en ocasiones, después de la "consulta", toma medicamento de la farmacia y lo entrega a los familiares de los migrantes para que se los envíen a
} EUA.
Estrategia 3: servicios privados

Cuando la enfermedad es grave o requiere atención de urgencia, algunos migrantes deciden utilizar servicios privados en EUA, asegurándose de buscar un profesional que no los delate ante las autoridades migratorias. Por lo regular acude con el médico por recomendación de otros migrantes que recibieron atención con anterioridad y cuya experiencia fue positiva. En virtud del costo y la barrera del idioma, no son muchos mexicanos indocumentados los que deciden utilizar servicios privados de salud. El surtimiento de los medicamentos se realiza casi siempre en farmacias privadas.

Se recogió el siguiente testimonio en grupos focales:

A mi hermana le cobraron 600 dólares porque se le infectó una muela y no se la atendió, (...) ya después se atendió y se la operaron y le cobraron todo eso. De un dedo también la operaron y le salió bien caro (GF P:6)

Segundo escenario: migrantes enfermos que se trasladan a territorio mexicano

Este escenario incluye la necesidad de someterse a un procedimiento quirúrgico o la vigilancia de problemas crónicos. En el escenario se identificaron dos estrategias que implican la necesidad de trasladarse a México (figura 2).

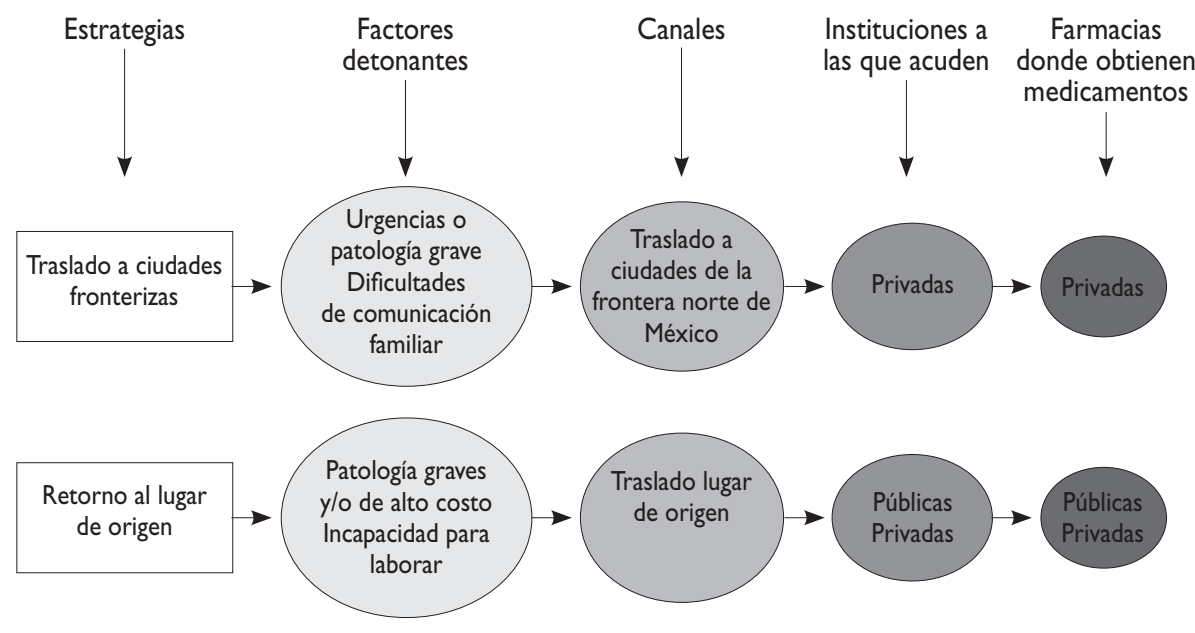

Fuente: elaboración propia a partir de los datos del trabajo de campo realizado entre mayo de 2006 y abril de 2007

El estudio inició en junio del 2005 y concluyó en abril del 2008

Figura 2. Segundo escenario. Migrantes enfermos QUe Se trasladan a terRitorio mexicano 
Estrategia 4: traslado a ciudades fronterizas

El costo elevado de los fármacos y la necesidad de mostrar una receta médica en farmacias de EUA obligan a los migrantes a trasladarse a ciudades fronterizas.

Yo tengo un hermano que tiene diabetes. Allá le cuesta más cara la medicina, entonces se viene a Tijuana a comprar medicamento porque está más barato (GF P:7)

El traslado a las ciudades fronterizas incluye las consultas médicas y la compra de medicamentos. La gran demanda de los fármacos proveniente del lado norteamericano ha generado un importante incremento del número de farmacias en ciudades de este lado de la frontera. Otros aspectos que promueven el traslado es la presencia de enfermedades que exigen atención de urgencia, además de las dificultades de comunicación con familiares en México. No obstante, testimonios obtenidos en grupos focales señalan como la principal dificultad para trasladarse a ciudades fronterizas, el riesgo de ser detenido por la policía migratoria estadounidense.

Según lo que nos comentan es muy difícil la atención médica allá, los que no tienen seguro se aguantan o tienen que bajar a alguna frontera. Muchos migrantes no tienen la documentación, entonces es difícil que vengan a atenderse aquí en México y luego regresar a los Estados Unidos (GF P:10).

Estrategia 5: retorno al lugar de origen

Se decide por esta opción en particular cuando el migrante enfrenta algún problema de salud que implica incapacidad para trabajar, la existencia de una enfermedad grave o de costo elevado. Al respecto, un migrante que participó en uno de los grupos focales comentó su experiencia.

Andaba trabajando en un tráiler cargando tomates y a la hora de bajar una caja se atoró un brazo y se zafó un hueso. Cuando venía de regreso apenas por Guadalajara, con el frío ya me empezó a pegar más el dolor y el dinerito que gané allá se me acabó en curarme (GF P:3)

En otros casos, a pesar de disponer de documentos migratorios en regla que facilitan la atención, la dificultad para acceder a los servicios de salud en EUA obliga a los migrantes a regresar para ser atendidos

Yo tengo una hermana que se enfermó, ella tiene papeles, pero fue a que la atendieran y no pudo. Tuvo que venir a Michoacán a un hospital particular, gastó como 20 mil pesos. Allá nunca la atendieron. (GF P:12)

En situaciones en las que los migrantes enfrentan problemas de salud graves, como cáncer, tuberculosis o $\mathrm{VIH} /$ sida, también prefieren regresar a sus lugares de origen para recibir atención médica, por lo general en instituciones públicas. Personal médico manifestó que éstos son problemas de salud pública, con escaso control, y que particularmente los casos de VIH se han incrementado. En un grupo focal uno de los informantes comentó:

En la garita un muchacho se regresó de los Estados Unidos porque tenía sida, pero se vino demasiado tarde y murió (GF P:4)

Tercer escenario: migrantes que acuden a servicios de salud durante sus visitas a México

Estrategia 6: atención en su comunidad de origen durante visitas cortas

Tanto los migrantes como sus familiares y el personal de salud de las localidades consideradas en el estudio coincidieron ampliamente en que durante las visitas de los migrantes existe un incremento del uso de los servicios de salud, públicos y privados, en especial para acciones de prevención, tratamiento de problemas agudos y control de problemas crónicos (figura 3).

Básicamente vienen por enfermedades respiratorias, por el cambio del clima y porque les gusta llegar a comer comida mexicana y les dan problemas estomacales (P:9)

Los que hemos estado en el primer nivel de atención, ya sabemos que en noviembre, diciembre y enero se nos dobla la consulta porque viene toda la familia y pasan al Centro de Salud. Aumentan algunos servicios, como planificación familiar y estudios de laboratorio (P:10).

Algunas unidades médicas han implementado mecanismos de atención preventiva para la captación de la población migrante y sus familiares en sus periodos de retorno.

En noviembre revisamos a todos los esposos migrantes, les brindamos vacunas, o sea, protección preventiva y les mandamos a hacer la prueba para detectar VIH (P:8)

\section{Discusión}

Los resultados expuestos en este documento presentan limitaciones inherentes al diseño de la investigación ba- 


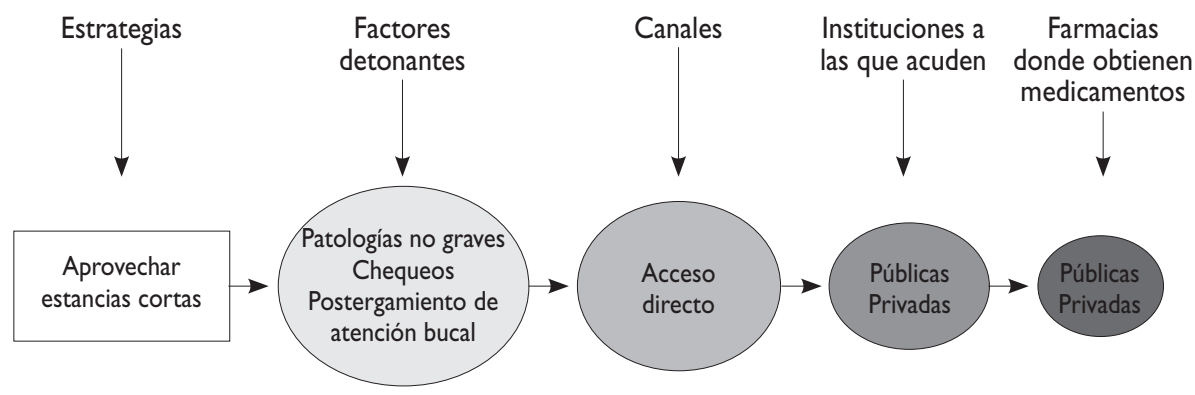

Fuente: elaboración propia a partir de los datos del trabajo de campo realizado entre mayo de 2006 y abril de 2007

El estudio inició en junio del 2005 y concluyó en abril del 2008

Figura 3.Tercer escenario. Migrantes Que acuden a servicios de salud durante sus visitas a México

sada en la recolección de información cualitativa. Dado que la muestra de informantes no es probabilística, los resultados no pueden generalizarse a la población de migrantes mexicanos en Estados Unidos de América. No obstante, la investigación proporciona datos valiosos de fuentes primarias que permiten indagar con mayor profundidad las opiniones recabadas. El presente trabajo describe algunas formas de acceso a la atención médica por parte de migrantes no informadas con anterioridad en las publicaciones especializadas.

La población migrante enfrenta múltiples y crecientes dificultades para acceder a los servicios de salud en EUA, relacionadas tanto con el contexto socioeconómico en el que se desarrollan como con las características de la oferta de servicios a ambos lados de la frontera. La investigación identifica que en esa nación, entre las principales dificultades para recibir servicios de salud, se encuentran la falta de aseguramiento y el temor a ser deportado por la condición de indocumentado. ${ }^{16,17}$

Es importante señalar que para los migrantes mexicanos los sucesos exógenos y endógenos respecto del campo de la salud, como la aplicación de políticas oficiales segregadoras contra los migrantes a niveles nacional y estatal (p. ej., muro en la frontera), la actividad violenta de grupos racistas, los recortes de recursos a los servicios públicos, el elevado costo de los servicios privados de salud y la barrera del idioma, crean un ambiente hostil que puede tener consecuencias negativas sobre su salud. Por otra parte, los programas de salud del gobierno estadounidense destinado a la población desprotegida no incluyen a los inmigrantes indocumentados (p. ej., Medicare, Medicaid). ${ }^{18-20}$
Ante la falta de aseguramiento para la salud de estas poblaciones, el estudio identificó seis grandes estrategias que la población utiliza para disponer de servicios de salud agrupadas en tres escenarios: a) atención médica de los migrantes en EUA, b) traslado a ciudades fronterizas y c) búsqueda de atención en épocas de visita a México. Las modalidades descritas son variadas e incluyen desde el consumo de remedios caseros e informales en ese país hasta la búsqueda de opciones de atención a larga distancia a través de la familia y los centros de salud del sistema mexicano.

Las formas descritas para la búsqueda de atención a la salud ponen en evidencia la dificultad para acceder a servicios formales de salud tanto en Estados Unidos de América como en México. Si bien en el primer país la dificultad de acceso a servicios está íntimamente vinculada por la falta de aseguramiento, en México un peso mayor lo tiene la baja capacidad de respuesta del sistema de salud en las comunidades de origen.

Uno de los elementos que se destacan de los resultados es el autoconsumo de fármacos. Este fenómeno ya ha sido analizado por otros autores, quienes documentan la autoprescripción de medicinas de patente y el uso de plantas medicinales y remedios caseros. En este sentido, cabe señalar el consumo indiscriminado de fármacos que se venden sin control en las ciudades fronterizas mexicanas. La venta transfronteriza de medicamentos ha experimentado un crecimiento. En consecuencia, se calcula que en 2005 se encontraban establecidas en Tijuana más de 1700 farmacias, mientras que del otro lado de la frontera, en San Diego, ese tipo de establecimientos no sumaba más de $100 .{ }^{21}$ Estos medicamentos son consumidos por mexicanos residentes, indocumentados ${ }^{22}$ 
y blancos no latinos nacidos en Estados Unidos. ${ }^{23}$

No existen referencias basadas en la realización de llamadas telefónicas a familiares en México para plantear el problema de salud y devolver la respuesta médica al inmigrante que se encuentra en Estados Unidos. Es probable que se trate de un mecanismo que se ha desarrollado a partir de la intensificación de las barreras físicas para el paso de migrantes ilegales y una mayor vigilancia en la frontera.

Es claro que la práctica de "consultar" y prescribir fármacos a larga distancia contraviene lo que establecen las normas oficiales mexicanas correspondientes. Por otra parte, es ilegal trasladar medicamentos no prescritos al territorio de EUA a través de la frontera, si bien el fenómeno ha crecido de manera considerable. La Food and Drug Administration (FDA) ha reconocido que más de dos millones de paquetes con fármacos entran al año por correo a Estados Unidos desde México. ${ }^{21}$

Dos de las medidas planteadas por los informantes para atender problemas de salud implican gastos considerables. Si se acude a servicios privados en EUA y se paga del bolsillo el gasto puede ser catastrófico, sobre todo en casos de urgencias o una enfermedad grave. Por otra parte, el retorno a México para enfrentar el problema implica gastos de traslado, medicamentos y pago de los servicios. Sin duda, los migrantes entrevistados prefieren regresar a México, en particular a la frontera, que sufragar el costo de los servicios en EUA. Este fenómeno es más común entre mexicanos que residen en ciudades o condados fronterizos, ya que los gastos de transporte se reducen en gran medida. ${ }^{24,25}$

\section{Conclusión}

La situación que viven los migrantes mexicanos indocumentados en Estados Unidos pone en evidencia su condición de vulnerabilidad, en especial al momento de buscar opciones de atención ante un problema de salud. En Estados Unidos, los mecanismos formales de atención son sumamente restrictivos y los riesgos percibidos de ser deportados vuelven aún más complicada la búsqueda. Se recurre de manera notoria a las relaciones familiares para obtener atención del sistema de salud en México, para lo cual se utilizan incluso mecanismos no convencionales de atención. Éste puede ser el escenario que millones de mexicanos sin documentos migratorios viven de manera cotidiana en aquella nación. Por lo tanto, se considera que es de vital importancia el desarrollo de programas de alto impacto para estas comunidades. Si bien los programas existentes hasta la fecha han tenido logros importantes, se requiere un esfuerzo articulado de gran envergadura para ofrecer una respuesta a gran escala, de acuerdo con la dimensión del fenómeno. Los esfuerzos por ofrecer aseguramiento y servicios coordinados a ambos lados de la frontera pueden representar un futuro promisorio para la salud de los migrantes mexicanos. ${ }^{26}$

\section{Agradecimientos}

El material que permitió redactar el artículo forma parte de un estudio más amplio, El Seguro Popular de Salud para familias con migrantes en Estados Unidos, realizado en Funsalud y el Instituto Nacional de Salud Pública durante los años 2005 y 2006, gracias al financiamiento del CONACYT. Parte del estudio se efectuó desde la Fundación Mexicana para la Salud. Agradecemos también a Elizabeth Aguilar, Iván Gómez y Mariel Rouvier por su participación.

\section{Referencias}

I. Durand J, Massey DS. Clandestinos. Migración México-Estados Unidos en los albores del siglo XXI. México: Miguel Ángel Porrúa y Universidad Autónoma de Zacatecas, 2003.

2. CONAPO [home page]. México: CONAPO [Consultado 2008 febrero 16]. La migración México-Estados Unidos. Disponible en: http://www. conapo.gob.mx/publicaciones/intensidadmig/capol.pdf

3. El Colegio de la Frontera Norte [home page]. México: Colegio de la Frontera Norte [Consultado 2009 mayo I2]. Cálculo sobre la base de diferentes fuentes de datos de México y Estados Unidos: Disponible en: http://www.colef.mx/Investigacion/DetalleProyectoCompleto.asp?CualCo digoProyecto $=10 \&$ VerTodos $=0 \&$ VerTTema $=\mid \&$ TipoTema $=\mid \&$ Cual Registro $=613 \&$ TieneProyecto $=$

4. Zúñiga $E$, Castañeda $X$, Giorguili S,Wallace $S$. Inmigrantes mexicanos y centroamericanos en Estados Unidos, acceso a la salud. México: SSA, Iniciativa de Salud México-California, University of California, Center for Health Policy Research, 2006.

5. Prentice JC, Pebley AR, Sastry N. Immigration status and health insurance coverage: who gains? who loses? Am J Pub Health 2005;95(I):109-116.

6. Frostin P.The impact of inmigration on health insurance coverage in the United States. EBRI notes 2005; 26 (6):2-8. http://www.ebri.org/pdf/ notespdf/EBRI_Notes_06-2005.pdf. [Consultado 2008 noviembre I5] 7. Shaw M, Dorling D, Smith G. Poverty, social exclusion and minorities. In: Marmot M,Wilkinson F (eds.). Social determinants of health. Oxford University, 1999:2I I-32.

8. Corvalan C, Driscoll T, Harrison J. Role of inmigrant factors in workrelated fatalities in Australia. JWork Envir 1994;20(5): 364-370.

9. Trovato F.Violent and accidental mortality among four immigrants groups in Canada. Soc Biol 1992;39(I):82-101.

10. Nigenda G, Ruiz JA. Servicios de salud y seguro popular para familias con migrantes. México: Instituto Nacional de Salud Pública de México, Fundación Mexicana para la Salud y CONACYT, 2008.

II. González M, González LM, Becker S, de la Sierra LA, York P, Gardner S, et al. Salud del migrante: propuesta de un seguro binacional de salud.

Cuernavaca: Instituto Nacional de Salud Pública, 2008.

12. Banco de México, Informe anual 2005, México, abril 2006.

13. Nigenda G, Lockett L, Manca C, Mora G. Non-biomedical health care practices in the State of Morelos: analysis of an emergent phenomenon. Sociol Health IIIn 200I;23; I:3-23. 
14. Consejo Nacional de Población. Índice de intensidad migratoria México-Estados Unidos, 2000. México: CONAPO, 2002: 194.

15. Tuirán R. Migración México-Estados Unidos: presente y futuro. Est Demog Urb 200I;16: 457- 462.

16. Hubbell FA, Waitzkin H, Mishra SI, Dombrink J, Chavez LR. Access to medical care for documented and undocumented latinos in a southern California county. West J Med I99|;154(4):414-417

17. Berk ML, Schur CL. The effect of fear on access to care among undocumented latino immigrants. I Immigr Health 200I; 3(3):I5I-I56. 18. Berk ML, Schur CL, Chavez LR , Frankel M. Health care use among undocumented latino immigrants is free health care the main reason why latinos come to the United States? A unique look at the facts. Health Affairs 2000; 19(4):51-64.

19. Carrasquillo O, Carrasquillo Al, Shea S. Health insurance coverage of immigrants living in the United States: differences by citizenship status and country of origin. Am J Public Health 2000;90(6):917-923.

20. Ortega AN, Fang H, Perez VH, Rizzo JA, Carter-Pokras O, Wallace SP, et al. Health care access, use of services, and experiences among undocumented mexicans and other latinos. Arch Intern Med 2007; I67(2I):2354-2360

21.Vargas J. El mercado transfronterizo de servicios de salud privados entre Tijuana, México y San Diego. Econ Mun 2005; 13:163-184.

22. Escobedo LG, Cardenas VM. Utilization and purchase of medical care services in Mexico by residents in the United States of America, 1998-1999. Rev Panam Salud Publica 2006; 19(5):300-305.

23. Wallace SP, Mendez-Luck C, Castañeda X. Heading South: mexican immigrants confront poor access to care in the US. EUA: California Center for Population Research, 2007.

24. Landeck M, Garza C, Mark Q. Utilization of physician health care services in Mexico by US hispanic border residents. Health 2002;20(I):3-16. 25. Macias EP, Morales LS. Poor underserved. Crossing the border for health care.J Health Care 200I;12(I):77-78.

26. Pardinas JE. Los retos de la migración en México. Un espejo de dos caras. México: CEPAL-Serie de Estudios y Perspectivas, No. 99, 2008. 